\title{
Accuracy of Cone Beam Computed Tomography for Periodontal Defect Measurements
}

\author{
Kelly A. Misch, ${ }^{*}$ Erica S. Yi, ${ }^{*}$ and David P. Sarment*
}

Background: Periodontal diagnosis relies heavily on traditional two-dimensional radiographic assessment. Despite efforts in improving reliability, current methods of detecting bone level changes over time or determining three-dimensional architecture of osseous defects are inadequate. To address these issues, computed tomography (CT) has been explored because of its ability to produce accurate three-dimensional imaging, but limitations such as radiation, machine size, and cost have made this approach impractical. Recently, cone beam computed tomography (CBCT) has turned this concept into potential reality because these lower-cost small machines produce high-quality data. Yet there is little research to establish periodontal bone measurement using CBCT as a valid method. Therefore, the aim of this study was to compare CBCT measurements of periodontal defects to traditional methods.

Methods: Artificial osseous defects were created on mandibles of dry skulls. CBCT scanning, periapical radiography (PA), and direct measurements using a periodontal probe were compared to an electronic caliper that was used as a standard reference.

Results: Linear measurements for all defects revealed no statistical differences between bone sounding, radiography, and CBCT. There was a significant difference when comparing isolated interproximal measurements using a probe versus the caliper $(P<0.001)$ but no significant difference for CBCT or radiography. All bony defects were identifiable and measurable directly or with CBCT. In comparison, buccal and lingual defects could not be measured with radiographs.

Conclusions: Overall, all three modalities are useful for identifying interproximal periodontal defects. Compared to radiographs, the three-dimensional capability of CBCT offers a significant advantage because all defects can be detected and quantified. J Periodontol 2006;77:1261-1266.

\section{KEY WORDS}

Alveolar bone loss; diagnosis; periodontal disease; radiographic image enhancement; three-dimensional imaging; $x$-ray computed tomography.

\footnotetext{
* Department of Periodontics and Oral Medicine, University of Michigan School of Dentistry Ann Arbor, MI.
}

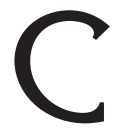
urrent approaches to diagnose periodontal disease include probing of gingival tissues and radiographs to evaluate osseous support. ${ }^{1}$ Imaging includes bitewings and periapical (PA) radiographs, ${ }^{2}$ with the main radiographic technique used for periodontal diagnosis being the paralleling extension cone method. . $^{3,4}$ Unfortunately, radiographic methods are severely limited by the inherent overlay of anatomic structures and the difficulty to reproduce angles over time. There is ample research demonstrating that funnel-shaped or lingually located defects cannot be detected ${ }^{5}$ and that destruction of the buccal plate can be undiagnosed or undistinguished from lingual defects. ${ }^{6}$ Further studies comparing radiographs to presurgical measurements concluded that bone loss can be underestimated by $\sim 1.5 \mathrm{~mm}$, with large variations between examiners. ${ }^{7}$ Consequently, traditional radiography remains a limited diagnostic tool. ${ }^{8}$

To address these issues, computed tomography (CT) has been explored because it enables cross-sectional and three-dimensional analysis without distortion. CT has been used for imaging of the temporomandibular joint, ${ }^{9}$ evaluating oral osseous lesions, ${ }^{10,11}$ assessing maxillofacial deformities, ${ }^{12}$ and preoperative planning of dental implants. ${ }^{13,14}$ Unfortunately, CT is impractical because of machine cost, complexity, high radiation, and relatively low resolution. 
More recently, cone beam computed tomography (CBCT) was introduced for head and neck applications. ${ }^{15-19}$ Contrary to CT, it consists of a conical radiographic source and a high-performance digital panel detector. In most CBCT machines, the apparatus is similar in size to a conventional panoramic machine, the examination takes $\sim 30$ seconds, and radiation is within range of an intraoral full-mouth series. ${ }^{20,21}$ In addition, CBCT resolution can be as small as $0.2 \mathrm{~mm}$, compared to 0.5 to $1 \mathrm{~mm}$ for CT. There is some preliminary evidence that it is reliable for evaluation measurements of large structures associated with dental and maxillofacial imaging, ${ }^{22}$ and it may be adequate to visualize periodontal alveolar bone changes, but little research has been performed. Therefore, the aim of the present study was to compare linear measurements of periodontal defects using CBCT to traditional methods.

\section{MATERIALS AND METHODS}

After approval from the University of Michigan Anatomic Donation Department was obtained, two human dry cadaver skulls with existing horizontal bone loss up to $20 \%$ were selected and used from January to July 2005. Infrabony buccal, lingual, and interproximal defects of varying width and height were created using a one-quarter-round dental bur in the mandibular premolar and molar regions. For each tooth, grooves were also placed vertically into the roots from the cemento-enamel junction (CEJ) to the apical base of the defect at each line angle (mesio-buccal, mesio-lingual, disto-buccal, and distolingual), mid-buccally and mid-lingually. Accessory gutta percha cones were superglued into the grooves to establish a reference point for probing, radiographic, and CBCT measurements. For each tooth under investigation, PA radiographs were taken using $\mathrm{F}$-speed film. The film was placed parallel to the specific tooth with the central ray of the $x$-ray device perpendicular to the tooth of interest.

CBCT scanning was performed using a clinically available machine. ${ }^{\dagger}$ Data were acquired at a medium resolution of $0.4 \mathrm{~mm}$, using 20 -second scanning (120 KVp and $47.74 \mathrm{mAs}$ ) to imitate current clinical methods (Fig. 1). Three examiners underwent a training session for probing techniques, radiographic measurements, and use of digital tools for CBCT. All measurements were performed blindly and repeated twice, with at least 1 week between sessions. Measurements of the same site, using two techniques, could not be performed during the same session. Direct measurements on the dry skull and for the PA radiographs were taken with one North Carolina periodontal probe. All PA films were mounted and visualized with a hand-held view box. The CBCT software had an electronic measuring tool to the nearest hun-
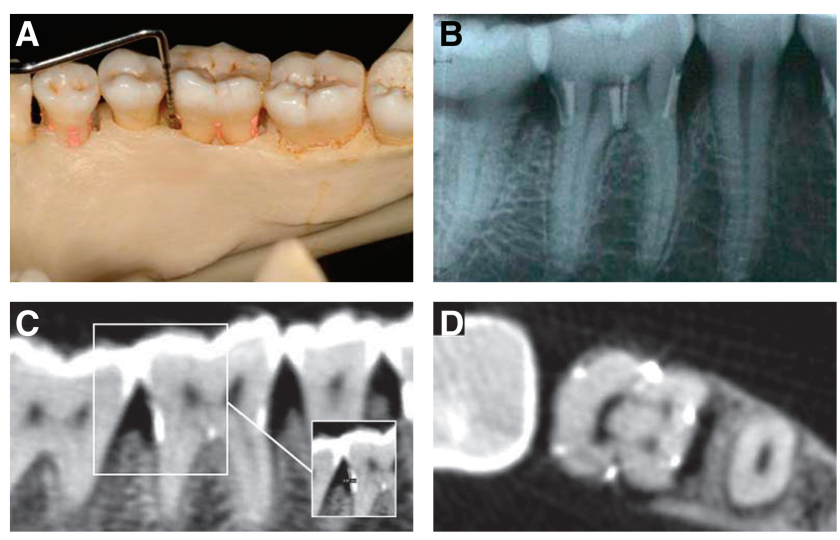

Figure 1 .

Periodontal measurements of osseous defects were obtained at reproducible locations using gutta-percha markers and a periodontal probe $(\boldsymbol{A})$, periapical radiographs $(\boldsymbol{B})$, and a digital ruler using cone beam computed tomography software (C). D) Note that landmarks were easily detected for reproducible location of measurements.

dredth of a millimeter. Cross-sectional slice thickness was reconstructed to $1 \mathrm{~mm}$ for all measurements, and each slice location was identified using the gutta percha location. All CBCT images were identical for each measurer as they were established by the principal investigator, saved, and loaded at each session. The examiners used only one computer, and screen settings remained unchanged. The examiners had the ability to change bone density and the size of the image to aid in optimal viewing.

Three measurements were taken for each defect (Fig. 2): 1) the length from the CEJ to the base of the bony defect $(A) ; 2$ ) the length from the CEJ to the crest of bone adjacent to the defect (B); and 3 ) the width of the defect $(C)$.

For gold-standard reference, all sites were injected with impression material. Height (A) and width (C) of each defect impression were measured and recorded with an electronic caliper. ${ }^{\ddagger}$ The accuracy of impression and caliper measurements was verified using another set of simulated defects of known height and width. These engineered cavities were prepared in a cast acrylic bloc using flute end mills $\S$ mounted in a vertical milling machine.l Drill diameter was \pm $0.05 \mathrm{~mm}$, and depth accuracy was $\pm 0.0254 \mathrm{~mm}$. Using the electronic caliper, each defect was measured blindly at two separate sessions.

\section{Statistical Analysis}

Statistical analysis was performed using statistical software. "l Coefficients of variation, using Pearson's

$\uparrow$ I-CAT, Imaging Sciences International, Hatfield, PA.

† Absolute Coolant Proof Caliper IP65, Mitutoyo Corporation, Kanagawa, Japan.

$\S$ Niagra Cutter, Amherst, NY.

I| Bridgeport Textron, Bridgeport, CT.

II SPSS version 14, SPSS, Chicago, IL. 


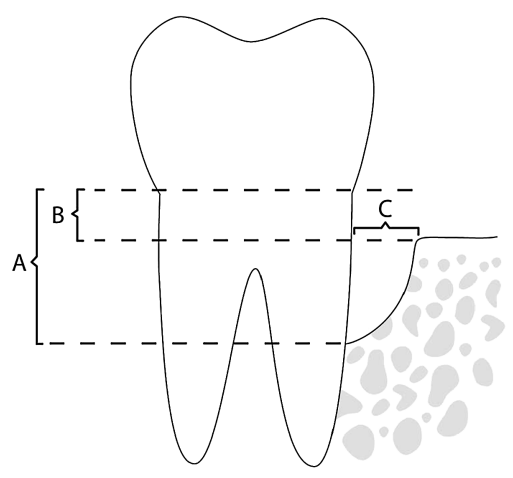

Figure 2.

Three study measurements were recorded for each site: A) height from the cemento-enamel junction to the apex of the defect; $B$ ) height of the defect; and C) width of the defect.

method, were calculated to determine variability within examiners and among techniques. Two-way analysis of variance was performed to compare groups. Finally, power analysis was calculated with a confidence level of $80 \%$.

Sample size was calculated using pilot data and assuming that means would remain $\leq 0.5 \mathrm{~mm}$. A $5 \%$ level of significance and an $80 \%$ power were used to calculate minimum sample sizes of $21 .^{23}$

\section{RESULTS}

Measurements were performed on all locations, except for 10 (33\%) buccal or lingual defects, using radiography because of lack of visualization. All infrabony defects were detected using CBCT and the probe. Statistical power was greater than 0.95 for all comparisons and varied from 0.95 to 0.99 . Correlations among examiners were calculated for all sites and modalities, with the exception of undetectable buccal and lingual defects on the radiographs. Correlations varied between 0.09 and 0.99 (Table 1 ), with an average of $0.4,0.53,0.62$, and 0.95 for direct, PA, CBCT, and impression measurements, respectively. Correlations between examiners were also calculated similarly. For the distance between the CEJ and apex of the defect, they were 0.41 , 0.84 , and 0.65 for direct, PA, and CBCT measurements, respectively. For defect width, they were $0.59,0.41$, and 0.64 for direct, PA, and CBCT measurements, respectively.

Height (distance A between cemento-enamel junction and apex of the defect) for each method was compared against the electronic caliper. Mean errors for all measurements were $0.41 \mathrm{~mm}( \pm 1.19 \mathrm{SD}), 0.27 \mathrm{~mm}$ ( $\pm 1.26 \mathrm{SD})$, and $0.34 \mathrm{~mm}( \pm 1.5 \mathrm{SD})$ for CBCT, PA radiographs, and probing, respectively. These results were not statistically different $(P>0.35)$ (Fig. 3 and Table 2). There was a statistically significant differ-
Table I.

\section{Correlations Within Examiners for Measurements A, B, and C}

\begin{tabular}{l|c|c|c|c|c}
\hline & & Probe & PA & CT & Caliper \\
\hline All measurements & A & 0.13 & 0.66 & 0.78 & 0.9 I \\
& B & 0.45 & 0.54 & 0.54 & NA \\
& C & 0.60 & 0.38 & 0.61 & 0.98 \\
\hline Interproximal only & A & 0.12 & 0.65 & 0.70 & 0.90 \\
& B & 0.32 & 0.54 & 0.66 & NA \\
& C & 0.63 & 0.38 & 0.66 & 0.98 \\
\hline Buccal and lingual only & A & 0.46 & NM & 0.86 & 0.98 \\
& B & 0.49 & NM & 0.32 & NA \\
& C & 0.38 & NM & 0.49 & 0.99 \\
\hline
\end{tabular}

$\mathrm{NA}=$ not applicable; $\mathrm{NM}=$ not measured because of insufficient data.

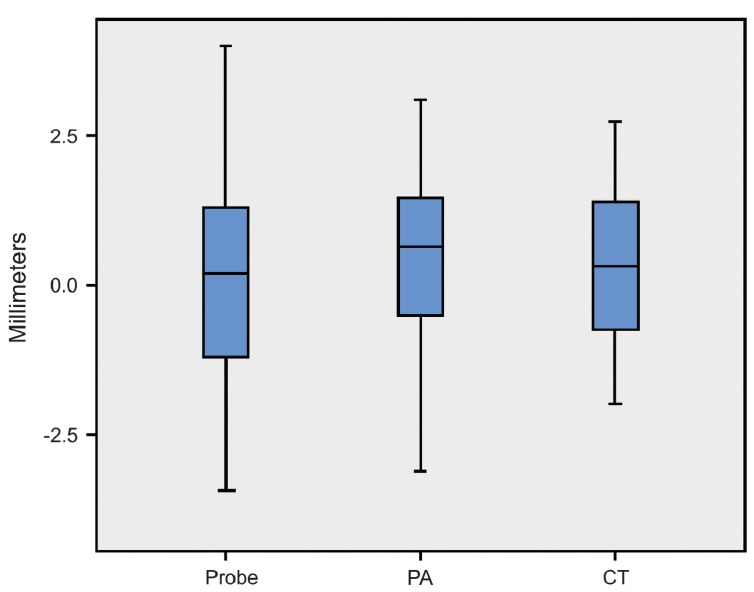

Figure 3.

Errors in height measurements (distance A) for all defects (median, first and third quartiles, and maximums and minimums). There was no statistically significant difference between the probe, PA radiographs (PA), and CT.

ence between examiners with regard to radiographs $(P=0.018)$.

With isolated interproximal results only, means were $0.36 \mathrm{~mm}( \pm 1.2 \mathrm{SD}), 0.37 \mathrm{~mm}( \pm 1.18 \mathrm{SD})$, and $0.60 \mathrm{~mm}( \pm 1.43 \mathrm{SD})$ for CBCT, PA radiographs, and probing, respectively. These results were statistically different for probing versus the other two methods $(P<0.001)$ but not different for CBCT versus PA radiographs $(P>0.5)$ (Fig. 4 and Table 2$)$. For interproximal measurements and for all methods, there was no statistical difference between examiners $(P>0.28)$. 
Table 2.

\section{Errors (means and SDs) From Gold Standard Using a Periodontal Probe, Radiographs (PA), or CBCT (CT)}

\begin{tabular}{|c|c|c|c|c|}
\hline & & Probe & PA & CT \\
\hline \multirow[t]{2}{*}{ All measurements } & Mean & 0.34 & 0.27 & 0.41 \\
\hline & SD & 1.50 & 1.26 & 1.19 \\
\hline \multirow[t]{2}{*}{ Interproximal only } & Mean & 0.60 & 0.37 & 0.36 \\
\hline & SD & 1.43 & 1.18 & 1.20 \\
\hline
\end{tabular}

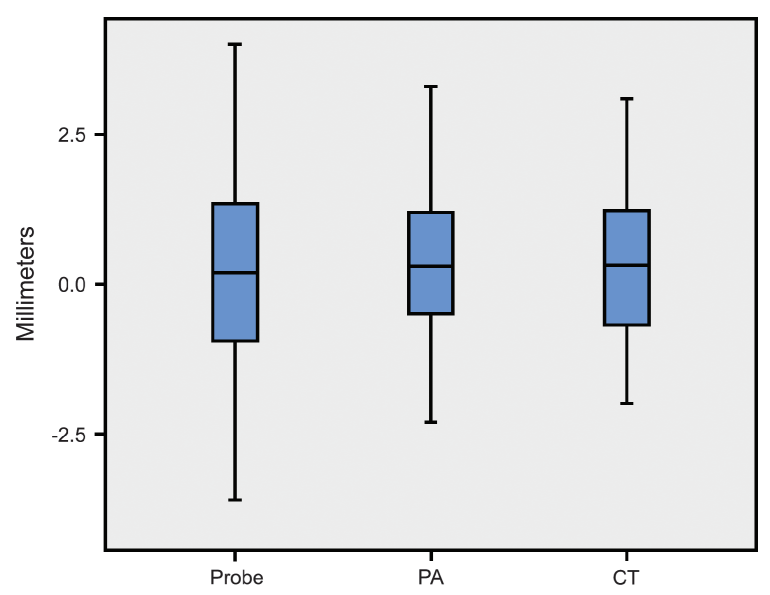

Figure 4.

Errors in height measurements (distance A) for interproximal defects only (median, first and third quartiles, and maximums and minimums). There was no statistically significant difference between the probe, PA radiographs (PA), and CT.

Measures of defect widths were recorded and compared to caliper measurements for direct and CBCT techniques. For radiographs, most buccal and lingual measures could not be performed, according to examiners, and only interproximal results were compared. For all sites combined, mean differences were $0.93 \mathrm{~mm}( \pm 0.65 \mathrm{SD})$ and $1 \mathrm{~mm}( \pm 0.67 \mathrm{SD})$ for direct and CBCT measures, respectively. For interproximal areas only, results were $1.14 \mathrm{~mm}( \pm 0.62$ $\mathrm{SD}), 1.16 \mathrm{~mm}( \pm 0.62 \mathrm{SD})$, and $1.03 \mathrm{~mm}( \pm 0.51$ $\mathrm{SD}$ ) for direct measures, CBCT measures, and radiographs, respectively. These means were not statistically different $(P>0.1)$. For buccal and lingual measures, the direct measurement mean difference was 0.53 ( $\pm 0.51 \mathrm{SD})$, and the CBCT mean difference was 0.7 ( $\pm 0.68 \mathrm{SD})$. These results were not statistically different $(P>0.09)$.

\section{DISCUSSION}

Potential use of CT for periodontal evaluation has been an area of interest in the past, recently becoming of actuality with the advent of new technology. Yet there has been little research to validate the use of recent CT methods for potential diagnosis of periodontal bone level. Within the limits of the study, the present results are evidence that CBCT is as accurate as direct measurements using a periodontal probe and as reliable as radiographs for interproximal areas. Because buccal and lingual defects could not be diagnosed with radiography, CBCT was a superior technique.

Using human cadaver jaws, Fuhrmann et al. ${ }^{10}$ also compared radiographs with CBCT and found that only $60 \%$ of infra-alveolar bony defects were identified on radiographs, whereas $100 \%$ could be distinguished using CBCT. This is similar to the results of the present study, also showing that all defects were detected using CBCT, but only $67 \%$ of them were diagnosed using radiography because buccal and lingual defects were not visible on the two-dimensional films. Their measurements resulted in a mean underestimation of $2.2 \mathrm{~mm}$ using radiographs and $0.2 \mathrm{~mm}$ using CBCT, which contrasts slightly with our study, where an overestimation of $0.27 \mathrm{~mm}$ for PA radiographs and 0.41 $\mathrm{mm}$ for CBCT was found. This variation between studies is likely due to methodology. It is interesting to note that 0.2 and $0.4 \mathrm{~mm}$ are the sizes of one and two voxels, the smallest detectable units utilized in these studies. Furthermore, the periodontal probe used in the study for measuring radiographs imitates the clinical setting but is a potential limitation: digital radiographs and digital measuring techniques may render more accurate results, although they would not change the inability to measure in the buccal and lingual direction.

Using animal and human mandibles, Mengel et al. ${ }^{24}$ compared periapical radiographs, panoramic films, CT and CBCT measurements of periodontal defects (fenestrations, dehiscences, and furcations) to their corresponding histologic specimens. Intraoral radiography was limited by visibility in the buccolingual direction, and image quality (contrast, brightness, distortion, overlay, clarity of bone structures, and focus) was superior using CBCT. When using radiographs, they reported mean height discrepancies (0.29 $\mathrm{mm})$ comparable to the present study $(0.27$ $\mathrm{mm})$. CBCT mean height was 0.16 versus $0.41 \mathrm{~mm}$ in the present report. Again, methods may account for these minimal differences. As expected, similar outcomes were reported among other studies when evaluating buccal and lingual defects only ${ }^{25}$ or when comparing CT with other radiographic methods such as cephalometry. ${ }^{26}$

Although two-dimensional radiography is of use for interproximal lesions, ${ }^{6}$ its limitation was anticipated 
during early investigations determining its diagnostic value for periapical and periodontal disease. ${ }^{27,28}$ On the other hand, geometric accuracy was expected for CBCT: using an in vitro geometric model to test a head and neck CBCT, Marmulla et al. ${ }^{29}$ found that variation was $0.13 \mathrm{~mm}( \pm 0.09 \mathrm{SD})$ with a maximum deviation of $0.3 \mathrm{~mm}$; using large measurements of skulls in vitro with the same machine, Lascala et al. ${ }^{22}$ found errors varying from 0.07 to $0.2 \mathrm{~mm}$. The present results are not as accurate because of human intervention in detecting bone level. Furthermore, gutta percha was placed on roots adjacent to measuring sites to standardize measurement localizations. Visualization of gutta percha on radiographic images also facilitated identification of CEJs, which would be more difficult in a clinical setting, resulting in a potential error increase. In contrast, using a dry skull with artificial defects may have influenced results because radiographic and CT representation of cortical outline was missing, thus affecting bone-level detection negatively.

Despite these encouraging results, CT has been scrupulously limited to advanced dental applications such as maxillofacial trauma or complex implant treatments because of higher radiation doses. ${ }^{30}$ Yet there is evidence that CBCT radiation is highly reduced compared to traditional CT. Comparing skin exposure of traditional CT versus a small-view CBCT, Honda et al. $^{31}$ reported a total radiation reduction from 160 to $1.19 \mathrm{mSv}$. Investigating organ exposure, Scaf et al. ${ }^{32}$ reported a radiation exposure of 1.031 $\mathrm{mSv}$ for a maxilla and $2.426 \mathrm{mSv}$ for a mandible using CT. This is compared to a reported CBCT organ radiation of $0.037 \mathrm{mSv}$ versus a panoramic film that was $0.022 \mathrm{mSv}$ in one study ${ }^{21}$ or to $0.15 \mathrm{mSv}$ for a full-mouth series. ${ }^{33}$ Finally, it is likely that radiation exposure will further decrease as technology evolves.

\section{CONCLUSIONS}

CBCT measurements compared well to traditional methods, with the advantage of allowing observance of periodontal defects in all directions. The present research calls for further clinical investigation to confirm that this method is widely applicable for use in periodontal diagnosis.

\section{ACKNOWLEDGMENTS}

The authors thank Drs. Dyda, Jabero, and Pinsky of the University of Michigan School of Dentistry. We also thank Anna Shafto, Department of Periodontics and Oral Medicine, University of Michigan School of Dentistry, for review of the manuscript. This study was supported by the Michigan Clinical Oral Health Research grant.

\section{REFERENCES}

1. Jeffcoat MK. Current concepts in periodontal disease testing. J Am Dent Assoc 1994;125:1071-1078.
2. Molander B. Panoramic radiography in dental diag nostics. Swed Dent J Suppl 1996;119:1-26.

3. Jeffcoat MK. Radiographic methods for the detection of progressive alveolar bone loss. J Periodontol 1992; 63(Suppl.):367-372.

4. Reddy MS. Radiographic methods in the evaluation of periodontal therapy. J Periodontol 1992;63(Suppl.): 1078-1084.

5. Ramadan AB, Mitchell DF. A roentgenographic study of experimental bone destruction. Oral Surg Oral Med Oral Pathol 1962;15:934-943.

6. Rees T, Biggs NL, Collins CK. Radiographic interpretation of periodontal osseous defects. Oral Surg Oral Med Oral Pathol Oral Radiol Endod 1971;2:141-153.

7. Eickholz P, Hausmann E. Accuracy of radiographic assessment of interproximal bone loss in intrabony defects using linear measurements. Eur J Oral Sci 2000;108:70-73.

8. Hirschmann PN. Radiographic interpretation of chronic periodontitis. Int Dent J 1987;37:3-9.

9. Honda K, Arai Y, Kashima M, et al. Evaluation of the usefulness of the limited cone-beam CT (3DX) in the assessment of the thickness of the roof of the glenoid fossa of the temporomandibular joint. Dentomaxillofac Radiol 2004;33:391-395.

10. Fuhrmann RA, Bucker A, Diedrich PR. Assessment of alveolar bone loss with high resolution computed tomography. J Periodontal Res 1995;30:258-263.

11. Ida M, Watanabe H, Tetsumura A, Kurabayashi T. CT findings as a significant predictive factor for the curability of mandibular osteomyelitis: Multivariate analysis. Dentomaxillofac Radiol 2005;34:86-90.

12. Hamada Y, Kondoh T, Noguchi K, et al. Application of limited cone beam computed tomography to clinical assessment of alveolar bone grafting: A preliminary report. Cleft Palate Craniofac J 2005;42:128-137.

13. Casap N, Tarazi E, Wexler A, Sonnenfeld U, Lustmann J. Intraoperative computerized navigation for flapless implant surgery and immediate loading in the edentulous mandible. Int J Oral Maxillofac Implants 2005; 20:92-98.

14. Parel SM, Triplett RG. Interactive imaging for implant planning, placement, and prosthesis construction. J Oral Maxillofac Surg 2004;62(Suppl. 2):41-47.

15. Arai Y, Tammisalo E, Iwai K, Hashimoto K, Shinoda K. Development of a compact computed tomographic apparatus for dental use. Dentomaxillofac Radiol 1999; 28:245-248.

16. Mozzo P, Procacci C, Tacconi A, Martini PT, Andreis IA. A new volumetric CT machine for dental imaging based on the cone-beam technique: Preliminary results. Eur Radiol 1998;8:1558-1564.

17. Araki K, Maki K, Seki K, et al. Characteristics of a newly developed dentomaxillofacial $\mathrm{x}$-ray cone beam CT scanner (CB MercuRay): System configuration and physical properties. Dentomaxillofac Radiol 2004;33:51-59.

18. Hashimoto K, Arai Y, Iwai K, Araki M, Kawashima S, Terakado M. A comparison of a new limited cone beam computed tomography machine for dental use with a multidetector row helical CT machine. Oral Surg Oral Med Oral Pathol Oral Radiol Endod 2003;95:371-377.

19. Danforth RA. Cone beam volume tomography: A new digital imaging option for dentistry. J Calif Dent Assoc 2003;31:814-815.

20. Sukovic P. Cone beam computed tomography in craniofacial imaging. Orthod Craniofac Res 2003; 6(Suppl. 1):31-36. 
21. Ludlow JB, Davies-Ludlow LE, Brooks SL. Dosimetry of two extraoral direct digital imaging devices: NewTom cone beam CT and Orthophos Plus DS panoramic unit. Dentomaxillofac Radiol 2003;32:229-234.

22. Lascala CA, Panella J, Marques MM. Analysis of the accuracy of linear measurements obtained by cone beam computed tomography (CBCT-NewTom). Dentomaxillofac Radiol 2004;33:291-294.

23. Cohen J. Statistical Power Analysis for the Behavioral Sciences, 2nd ed. Hillsdale, NJ: Lawrence Erlbaum; 1988:8-41.

24. Mengel R, Candir M, Shiratori K, Flores-de-Jacoby L. Digital volume tomography in the diagnosis of periodontal defects: An in vitro study on native pig and human mandibles. $J$ Periodontol 2005;76: 665-673.

25. Fuhrmann R. Three-dimensional interpretation of alveolar bone dehiscences: An anatomical-radiological study - Part I. J Orofac Orthop 1996;57:62-74.

26. Fuhrmann R. Three-dimensional interpretation of labiolingual bone width of the lower incisors. Part II. J Orofac Orthop 1996;57:168-185.

27. Bender IB, Seltzer S. Roentgenographic and direct observations of experimental lesions in bone. J Am Dent Assoc 1961;62:152-160.

28. Lee SJ, Messer H. Radiographic appearance of artificially prepared periapical lesions confined to cancellous bone. Int Endod J 1986;19:64-72.
29. Marmulla R, Wortche R, Muhling J, Hassfeld S. Geometric accuracy of the NewTom 9000 cone beam CT. Dentomaxillofac Radiol 2005;34:28-31.

30. Tyndall AA, Brooks SL. Selection criteria for dental implant site imaging: A position paper of the American Academy of Oral and Maxillofacial Radiology. Oral Surg Oral Med Oral Pathol Oral Radiol Endod 2000; 89:630-637.

31. Honda K, Arai Y, Iwai K, Hashimoto K, Saitou T, Shinoda K. Fundamental efficiency of new-style limited cone-beam CT (3DX): Comparison with helicalCT. Jpn J Tomogr 2001;27:193-198.

32. Scaf G, Lurie AG, Mosier KM, Kantor ML, Ramsby GR, Freedman ML. Dosimetry and cost of imaging osseointegrated implants with film-based and computed tomography. Oral Surg Oral Med Oral Pathol Oral Radiol Endod 1997;83:41-48.

33. Avendanio B, Frederiksen NL, Benson BW, Sokolowski TW. Effective dose and risk assessment from detailed narrow beam radiography. Oral Surg Oral Med Oral Pathol Oral Radiol Endod 1996;82:713-719.

Correspondence: Dr. David Sarment, Department of Periodontics and Oral Medicine, University of Michigan School of Dentistry, 1011 N. University Ave., Ann Arbor, MI 48109. Fax: 734/763-5503; e-mail: sarment@umich.edu.

Accepted for publication February 14, 2006. 